\title{
Statistical Model for the Orientation of Nonspherical Particles Settling in Turbulence
}

\author{
K. Gustavsson, ${ }^{1}$ J. Jucha ${ }^{2,3}$ A. Naso, ${ }^{4}$ E. Lévêque, ${ }^{4}$ A. Pumir, ${ }^{2}$ and B. Mehlig ${ }^{1}$ \\ ${ }^{1}$ Department of Physics, Gothenburg University, 41296 Gothenburg, Sweden \\ ${ }^{2}$ Laboratoire de Physique, Ecole Normale Supérieure de Lyon and CNRS, F-69007 Lyon, France \\ ${ }^{3}$ Projektträger Jülich, Forschungszentrum Jülich GmbH, D-52425 Jülich, Germany \\ ${ }^{4}$ LMFA, Ecole Centrale de Lyon and CNRS, F-69134 Ecully, France
}

(Received 11 February 2017; revised manuscript received 26 July 2017; published 19 December 2017)

\begin{abstract}
The orientation of small anisotropic particles settling in a turbulent fluid determines some essential properties of the suspension. We show that the orientation distribution of small heavy spheroids settling through turbulence can be accurately predicted by a simple Gaussian statistical model that takes into account particle inertia and provides a quantitative understanding of the orientation distribution on the problem parameters when fluid inertia is negligible. Our results open the way to a parametrization of the distribution of ice crystals in clouds, and potentially lead to an improved understanding of radiation reflection or particle aggregation through collisions in clouds.
\end{abstract}

DOI: 10.1103/PhysRevLett.119.254501

How nonspherical objects settle in a turbulent environment is a question highly relevant to several domains. An example is provided by very small ice crystals in clouds (size $\sim 100 \mu \mathrm{m}$ ), which grow through aggregation to form precipitation-size particles (size $\sim 1 \mathrm{~mm}$ ) [1-4]. The settling of plankton in the ocean [5-7] can induce patchiness of the population, thereby affecting mating, feeding, and predation [8]. In these problems, the orientational degrees of freedom clearly affect not only settling and collision properties, but also light reflection [9]. As a prerequisite to a description of these effects, this Letter provides an understanding of the orientation statistics of small spheroids settling in a turbulent environment based on a statistical model, under the assumption that fluid inertia can be neglected.

The interaction between turbulence and settling leads to intriguing phenomena, even in the simpler case of spherical particles. Maxey found that turbulence increases the settling speed of a single small particle [10,11]. Substantial progress was recently achieved in understanding how two spherical particles settling together move relative to each other and collide [12-16].

In a fluid at rest the orientation of slowly settling nonspherical particles is determined by weak torques induced by the inertia of the fluid set in motion by the moving particles. For a single, isolated particle in a quiescent fluid this effect is well understood [17-21]. Particle-particle interactions give rise to intricate rotational dynamics [21].

Turbulence affects the orientation of a settling particle through turbulent vorticity and strain. In the absence of

Published by the American Physical Society under the terms of the Creative Commons Attribution 4.0 International license. Further distribution of this work must maintain attribution to the author(s) and the published article's title, journal citation, and DOI. settling the dynamics is well understood [7,22-30]. Neglecting fluid inertia, the direct numerical simulations (DNS) of turbulence by Siewert et al. [31] demonstrated that settling induces a bias in the orientation distribution of the particles. The physical origin of this bias is not known, and it is not understood how it depends on the parameters of the problem: the turbulent Reynolds number, $\mathrm{Re}_{\lambda}$, the Stokes number (particle inertia), the gravitational acceleration, and the particle shape. Also, how significant are non-Gaussian, intermittent small-scale features of the turbulent flow [32], such as intense vortex tubes [2], in aligning the particles?

To answer these questions we analyze a statistical model for the orientation of small heavy spheroids settling in homogeneous isotropic turbulence, for parameters relevant to cloud physics, and compare with results based on the DNS of turbulence. Figure 1 shows the predicted bias in the
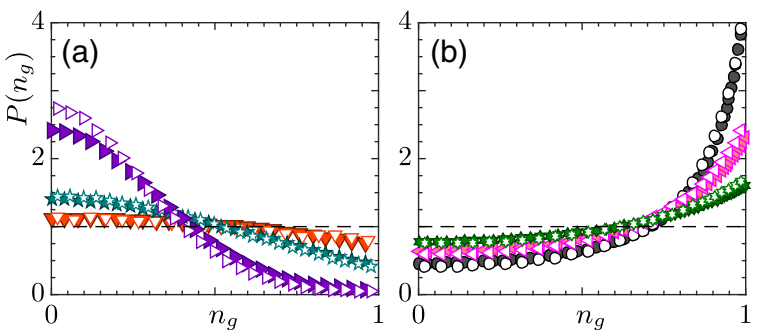

FIG. 1. Orientational bias of spheroids settling in turbulence. Distribution $P\left(n_{g}\right)$ of $n_{g} \equiv|\boldsymbol{n} \cdot \hat{\boldsymbol{g}}|$, with particle symmetry vector $\boldsymbol{n}$ and direction $\hat{\boldsymbol{g}}$ of gravity. (a) DNS results for $P\left(n_{g}\right)$ for oblate spheroids [aspect ratios $\lambda=0.01$ (inverted triangle), 0.02 (star), 0.05 (right-pointing triangle)]. Statistical-model simulations: open symbols. Dashed line: isotropic distribution $P\left(n_{g}\right)=1$. (b) Same, but for prolate spheroids: $\lambda=5$ (circle), 7.5 (left pointing triangle), 10 (star). Parameters: $\operatorname{Re}_{\lambda} \approx 95, F_{\mathrm{K}} \approx 70$, and $\mathrm{St}_{\mathrm{K}} \approx 4 \min (\lambda, 1 / \lambda)$ (see text); these correspond to values relevant to cloud physics, see the Supplemental Material [33]. 
distribution of the vector $\boldsymbol{n}$ pointing along the particle symmetry axis. The statistical-model predictions agree very well with the DNS results. This shows that non-Gaussian turbulent fluctuations are not important. The statistical model explains the sensitive parameter dependence of the DNS results. This is important because it allows us to parametrize the bias, to quantitatively understand the physical properties of the system. We analyze the model by an expansion in the "Kubo number" $\mathrm{Ku}$, a dimensionless correlation time of the flow [36]. Padé-Borel resummation yields excellent agreement with numerical simulations at $\mathrm{Ku}=0.1$, and qualitative agreement with DNS of turbulence. At larger $\mathrm{Ku}$ the theory fails to converge, but the model still qualitatively explains the underlying mechanisms. Finally, we discuss possible effects of fluid inertia.

Formulation of the problem.-The equations of motion for translation and rotation of a particle read

$m \ddot{\boldsymbol{x}}=\boldsymbol{f}+m g \hat{\boldsymbol{g}}, \quad \dot{\boldsymbol{n}}=\boldsymbol{\omega} \wedge \boldsymbol{n}, \quad(d / d t)[\mathbb{}(\boldsymbol{n}) \boldsymbol{\omega}]=\boldsymbol{T}$.

Here $g$ is the gravitational acceleration (direction $\hat{\boldsymbol{g}}$ ), $\boldsymbol{x}$ is the position of the particle, $\boldsymbol{n}$ its symmetry vector, $m$ its mass, $\boldsymbol{\omega}$ its angular velocity, and $\mathbb{J}(\boldsymbol{n})$ its inertia tensor in the lab frame. In the point-particle approximation, the force $f$ and torque $\boldsymbol{T}$ on a spheroid are $[26,37,38]$

$$
\left[\begin{array}{l}
\boldsymbol{f} \\
\boldsymbol{T}
\end{array}\right]=m \gamma\left[\begin{array}{ccc}
\mathbb{M}^{(t)} & 0 & 0 \\
0 & \mathbb{M}^{(r 1)} & \mathbb{M}^{(r 2)}
\end{array}\right]\left[\begin{array}{c}
\boldsymbol{u}-\boldsymbol{v} \\
\boldsymbol{\Omega}-\boldsymbol{\omega} \\
\mathbb{S}
\end{array}\right] .
$$

In Eq. (2), $\boldsymbol{v}$ is the particle velocity, $\boldsymbol{u}(\boldsymbol{x}, t)$ is the turbulent velocity field, $\boldsymbol{\Omega} \equiv \frac{1}{2} \boldsymbol{\nabla} \wedge \boldsymbol{u}$ is half the turbulent vorticity, $\mathbb{S}$ is the strain-rate matrix, the symmetric part of the matrix $\mathbb{A}$ of fluid-velocity gradients (its antisymmetric part is called $\mathbb{O}$ ), $\mathbb{M}^{(r)}$ and $\mathbb{M}^{(t)}$ are translational and rotational resistance tensors, $\mathbb{M}^{(t)} \equiv C_{\perp}^{(t)} \mathbb{1}+\left(C_{\|}^{(t)}-C_{\perp}^{(t)}\right) \boldsymbol{n} \boldsymbol{n}^{\top}, \mathbb{M}^{(r 1)} \equiv$ $K_{\perp}^{(r 1)} \mathbb{1}+\left(K_{\|}^{(r 1)}-K_{\perp}^{(r 1)}\right) \boldsymbol{n} \boldsymbol{n}^{\top}$, and $\mathbb{M}^{(r 2)}$ is a third-rank tensor. Explicit expressions for the $C$ and $K$ coefficients and for the third-rank tensor are known for spheroidal particles, as functions of the particle aspect ratio $\lambda$. See Supplemental Material (SM) [33]. Also crystals with discrete rotational symmetry have the tensorial structure of Eq. (2) [39], but the values of the $C$ and $K$ coefficients are not known in general.

For a fore-aft symmetric particle, the equations of motion (1) and (2) are invariant under $\boldsymbol{n} \rightarrow \boldsymbol{- n}$, so that only the magnitude $n_{g} \equiv|\boldsymbol{n} \cdot \hat{\boldsymbol{g}}|$ can play a role in the dynamics. The parameter $\gamma \equiv 9 \nu \rho_{f} /\left(2 a_{\|} a_{\perp} \rho_{p}\right)$ is the Stokes damping rate, $\nu$ is the kinematic viscosity of the fluid, $\rho_{f}$ and $\rho_{p}$ are fluid and particle mass densities, $2 a_{\|}$is the length of the particle symmetry axis, and $2 a_{\perp}$ is its diameter.

Our DNS of turbulence use the code described in Ref. [40] and in the SM [33]. The Kolmogorov scales $u_{\mathrm{K}}, \eta_{\mathrm{K}}$, and $\tau_{\mathrm{K}}$ are determined by the dissipation rate $\varepsilon \equiv$ $\nu\left\langle\operatorname{Tr} \mathbb{A} \mathbb{A}^{\top}\right\rangle$ (the average is along steady-state Lagrangian trajectories), and by $\nu \approx 1 \times 10^{-5} \mathrm{~m}^{2} \mathrm{~s}^{-1}$ (air). The particle aspect ratio is $\lambda \equiv a_{\|} / a_{\perp}$. The simulations were done for spheroids of varying $\lambda$ and with the largest axis length, $\max \left(a_{\|}, a_{\perp}\right)=150 \mu \mathrm{m}$, much smaller than $\eta_{\mathrm{K}}$ for values of $\varepsilon$ pertaining to mixed-phase clouds (DNS: $\varepsilon \approx 1,16$, and $256 \mathrm{~cm}^{2} \mathrm{~s}^{-3}$ ). Particle inertia is measured by the Stokes number $S t_{\mathrm{K}} \equiv\left(\gamma \tau_{\mathrm{K}}\right)^{-1}$. The mass-density ratio is $R \equiv \rho_{p} / \rho_{f} \approx 1000$ (ice crystals in air), and the dimensionless gravity parameter is defined as $F_{\mathrm{K}} \equiv g \tau_{\mathrm{K}} / u_{\mathrm{K}}$. An alternative but equivalent parametrization is in terms of $S_{v} \equiv F_{\mathrm{K}} S t_{\mathrm{K}}[41]$.

Statistical model.-We represent the undisturbed turbulent flow in the vicinity of a particle (smaller than the smallest turbulent eddy size, $\eta_{\mathrm{K}}$ ) by an incompressible, homogeneous, isotropic Gaussian random velocity field $\boldsymbol{u}(\boldsymbol{x}, t)$ with zero mean, correlation length $\ell$, correlation time $\tau$, and rms speed $u_{0}$. Details are given in the SM [33]. The statistical model has three dimensionless parameters, $\mathrm{St} \equiv(\gamma \tau)^{-1}, F \equiv g \tau / u_{0}$, and the Kubo number $\mathrm{Ku} \equiv$ $u_{0} \tau / \ell$. Turbulence corresponds to the persistent limit of this statistical model, the limit of large Kubo numbers. In this limit, the dimensionless parameters St and $F$ map to $\mathrm{St}_{\mathrm{K}}$ and $F_{\mathrm{K}}$ as follows: $\mathrm{St}_{\mathrm{K}}=\sqrt{5} \mathrm{Ku}$ St and $F_{\mathrm{K}}=$ $[F /(5 \mathrm{Ku})] \ell / \eta_{\mathrm{K}}$. Here $\ell / \eta_{\mathrm{K}}$ is the ratio between the size of the dissipation range and the Kolmogorov length. In turbulence this ratio depends weakly on the Reynolds number $\operatorname{Re}_{\lambda}$ [42], $\ell=c \eta_{\mathrm{K}} \operatorname{Re}_{\lambda}^{1 / 2}$. For the data shown in Fig. 1 we have $\operatorname{Re}_{\lambda}=95$ (Fig. S1 in the SM [33] shows results for other values of $\mathrm{Re}_{\lambda}$ ). We find good agreement between the statistical-model results at large $\mathrm{Ku}$ and the DNS for $c \approx 1.3$. For $\mathrm{Ku}>1$, the model predictions depend on two parameter combinations only [36], KuSt and $F / \mathrm{Ku}$. In terms of the DNS parameters this means that the orientation bias depends only on $\mathrm{St}_{\mathrm{K}}$ and $F_{\mathrm{K}} \mathrm{Re}_{\lambda}^{-1 / 2}$.

Perturbation theory.-Equations (1) and (2) are solved by expansion in powers of $\mathrm{Ku}[36,43]$. We outline the essential steps below; details are given in the SM [33]. We use dimensionless variables $t^{\prime} \equiv t / \tau, \boldsymbol{r}^{\prime} \equiv \boldsymbol{r} / \ell, \boldsymbol{u}^{\prime} \equiv \boldsymbol{u} / u_{0}$, and drop the primes. To calculate the steady-state distribution of $n_{g} \equiv|\boldsymbol{n} \cdot \hat{\boldsymbol{g}}|$ we must evaluate the fluctuations of the fluid-velocity gradients along particle paths. In the statistical model this is achieved by an expansion in $\delta x_{t} \equiv$ $\boldsymbol{x}_{t}-\boldsymbol{x}_{t}^{(d)}$ around the stationary deterministic solution of Eqs. (1), (2) for $\boldsymbol{u}=0$, namely, $\boldsymbol{x}_{t}^{(d)}=\boldsymbol{x}_{0}+\boldsymbol{v}_{s}\left(\boldsymbol{n}_{0}\right) t$ with settling velocity

$$
\boldsymbol{v}_{s}\left(\boldsymbol{n}_{0}\right)=F \mathrm{St}\left(\frac{\mathbb{1}}{C_{\perp}^{(t)}}+\frac{\boldsymbol{n}_{0} \boldsymbol{n}_{0}^{\top}}{C_{\|}^{(t)}}-\frac{\boldsymbol{n}_{0} \boldsymbol{n}_{0}^{\top}}{C_{\perp}^{(t)}}\right) \hat{\boldsymbol{g}},
$$

describing a straight but inclined settling path.

Expansions in $\delta \boldsymbol{x}_{t}$ yield power-series approximations for physical observables in powers of $\mathrm{Ku}$ [36]. To order $\mathrm{Ku}^{2}$ we find for $\boldsymbol{n}_{t}$ (details are given in the SM [33]) 


$$
\begin{aligned}
\boldsymbol{n}_{t}= & \boldsymbol{n}_{0}+\left.\mathrm{Ku} \int_{0}^{t} d t_{1}\left(1-e^{\left(t_{1}-t\right) C_{\perp}^{(r 1)} / \mathrm{St}}\right)\left(\delta \boldsymbol{x}_{t_{1}} \cdot \nabla\right)\left(\mathbb{O}\left(\boldsymbol{x}, t_{1}\right) \boldsymbol{n}_{0}+\frac{\lambda^{2}-1}{\lambda^{2}+1}\left\{\mathbb{S}\left(\boldsymbol{x}, t_{1}\right) \boldsymbol{n}_{0}-\left[\boldsymbol{n}_{0} \cdot \mathbb{S}\left(\boldsymbol{x}, t_{1}\right) \boldsymbol{n}_{0}\right] \boldsymbol{n}_{0}\right\}\right)\right|_{\boldsymbol{x}=\boldsymbol{x}_{t_{1}}^{(\mathrm{d})}} \\
& +\mathrm{Ku} \int_{0}^{t} d t_{1}\left(1-e^{C_{\perp}^{(r 1)}\left(t_{1}-t\right) / \mathrm{St}}\right)\left(\mathbb{O}\left(t_{1}\right) \boldsymbol{n}_{0}+\frac{\lambda^{2}-1}{\lambda^{2}+1}\left\{\mathbb{S}\left(t_{1}\right) \boldsymbol{n}_{0}-\left[\boldsymbol{n}_{0}^{\top} \mathbb{S}\left(t_{1}\right) \boldsymbol{n}_{0}\right] \boldsymbol{n}_{0}\right\}\right) \\
& +\mathrm{Ku}^{2} \int_{0}^{t} d t_{1} \int_{0}^{t_{1}} d t_{2}\left[\boldsymbol{c}_{i j k l}^{(O O)}\left(\boldsymbol{n}_{0} ; t, t_{1}, t_{2}\right) O_{i j}\left(t_{1}\right) O_{k l}\left(t_{2}\right)+\text { similar terms, with } O O \text { replaced by } O S \text { and } S S\right] .
\end{aligned}
$$

Here the coefficient $C_{\perp}^{(r 1)}$ is defined as $C_{\perp}^{(r 1)} \equiv$ $5 K_{\perp}^{(r 1)} /\left(a_{\|}^{2}+a_{\perp}^{2}\right)$. In Eq. (4) we have used the short-hand notations $\mathbb{O}(t) \equiv \mathbb{O}\left(x_{t}^{(d)}, t\right)$ and $\mathbb{S}(t) \equiv \mathbb{S}\left(x_{t}^{(d)}, t\right)$.

There are two types of contributions to (4). First, the terms in Eq. (4) that do not involve $\delta \boldsymbol{x}_{t}$ [second and third lines of Eq. (4)] depend only on the history of the fluid-velocity gradients along the paths $\boldsymbol{x}_{t}^{(d)}$. We refer to these terms as the "history contribution". The $c$ coefficients contain at most five powers of $\boldsymbol{n}_{0}$, and one must sum over all tensor products allowed by symmetry (Einstein convention). Details are given in the SM [33]. Second, the first integral shown in Eq. (4) depends on $\delta \boldsymbol{x}_{t}$. It is therefore sensitive to how turbulence modifies the settling paths. We conclude that this contribution is affected by "preferential sampling" [36].

We determine the steady-state moments $\left\langle\left(\boldsymbol{n}_{t} \cdot \hat{\boldsymbol{g}}\right)^{p}\right\rangle_{\infty}$ by first calculating the moments $\left\langle\left(\boldsymbol{n}_{t} \cdot \hat{\boldsymbol{g}}\right)^{p}\right\rangle_{\boldsymbol{n}_{0}}$ conditional on the initial orientation $\boldsymbol{n}_{0}$, using Eq. (4) and the relation

$$
\begin{aligned}
\left\langle\left(\boldsymbol{n}_{t} \cdot \hat{\boldsymbol{g}}\right)^{p}\right\rangle_{\boldsymbol{n}_{0}}= & \left(\boldsymbol{n}_{0} \cdot \hat{\boldsymbol{g}}\right)^{p}+p \mathrm{Ku}\left(\boldsymbol{n}_{0} \cdot \hat{\boldsymbol{g}}\right)^{p-1}\left\langle\boldsymbol{n}_{t}^{(1)} \cdot \hat{\boldsymbol{g}}\right\rangle_{\boldsymbol{n}_{0}} \\
& +\frac{p}{2} \mathrm{Ku}^{2}\left(\boldsymbol{n}_{0} \cdot \hat{\boldsymbol{g}}\right)^{p-2}\left\langle 2\left(\boldsymbol{n}_{0} \cdot \hat{\boldsymbol{g}}\right)\left(\boldsymbol{n}_{t}^{(2)} \cdot \hat{\boldsymbol{g}}\right)\right. \\
& \left.+(p-1)\left(\boldsymbol{n}_{t}^{(1)} \cdot \hat{\boldsymbol{g}}\right)^{2}\right\rangle_{\boldsymbol{n}_{0}}
\end{aligned}
$$

where $\boldsymbol{n}_{t}^{(i)}$ is the coefficient of $\mathrm{Ku}^{i}$ in Eq. (4). Equation (5) is valid to order $\mathrm{Ku}^{2}$. We average over the fluid-velocity fluctuations as described in Ref. [36]. The moments are independent of the initial position $\boldsymbol{x}_{0}$ due to homogeneity of the flow. We expect that effects of the initial velocity $v_{0}$ and angular velocity $\omega_{0}$ decay exponentially, so that they do not affect the steady state. We therefore set both to zero. Only the $\boldsymbol{n}_{0}$ dependence matters. In this way we obtain expressions for $\left\langle\left(\boldsymbol{n}_{t} \cdot \hat{\boldsymbol{g}}\right)^{p}\right\rangle_{\boldsymbol{n}_{0}}$, which involve secular terms that increase linearly with time as $t \rightarrow \infty$. However, these terms must vanish since $\boldsymbol{n}_{t}$ is a unit vector. This condition yields a recursion relation for the steady-state averages $\left\langle(\boldsymbol{n} \cdot \hat{\boldsymbol{g}})^{p}\right\rangle_{\infty}$, independent of $\boldsymbol{n}_{0}$. This recursion is valid for arbitrary values of $G \equiv \mathrm{Ku} F \mathrm{St} / C_{\perp}^{(t)}$, and to order $\mathrm{Ku}^{0}$. Note that $G$ can be large even if $\mathrm{Ku}$ is small. We solve the recursion by a series expansion in small $G$,

$\left\langle(\boldsymbol{n} \cdot \hat{\boldsymbol{g}})^{2 p}\right\rangle_{\infty}=\frac{1}{2 p+1}+\sum_{i=1}^{\infty} \frac{G^{2 i} \sum_{j=1}^{i} p^{j} A_{j}^{(2 i)}(S t, \lambda)}{\prod_{k=1}^{i+1}(2 p+2 k-1)}$.
The coefficients $A_{j}^{(2 i)}(S t, \lambda)$ depend on the shape and inertia of the particle, but not on $G$ or $p$. From Eq. (6) we obtain the Fourier transform of the probability distribution of $n_{g}=|\boldsymbol{n} \cdot \hat{\boldsymbol{g}}|$. Inverse Fourier transformation yields the distribution. To order $G^{4}$ we find

$$
\begin{aligned}
P\left(n_{g}\right)= & 1+\frac{1}{4}\left(3 n_{g}^{2}-1\right) A_{1}^{(2)} G^{2}+\frac{1}{32}\left[2\left(1-n_{g}^{2}\right)\left(5 n_{g}^{2}-1\right) A_{1}^{(4)}\right. \\
& \left.\left.+1-18 n_{g}^{2}+25 n_{g}^{4}\right) A_{2}^{(4)}\right] G^{4}+\cdots .
\end{aligned}
$$

The lowest-order term corresponds to a uniform distribution of $\boldsymbol{n}_{t}$. The $G^{2}$ term, $A_{1}^{(2)}$, turns out to be negative for disks and positive for rods (see Fig. S2 in the SM [33]). This explains the bias seen in Fig. 1: disks tend to fall edge on, and rods settle tip first.

Padé-Borel resummation.-Now consider higher orders in the $G$ expansion. The coefficients increase rapidly with the order of the expansion. This indicates that the series (6) is asymptotically divergent and must be resummed. Figure 2 demonstrates that Padé-Borel resummation $[36,44]$ of the series yields excellent results. Shown are results from a resummation of (6) to order $G^{34}$ (thick solid lines). These results agree very well with numerical simulations of the statistical model for $\mathrm{Ku}=0.1$ and $\mathrm{St}=10$ (symbols). The resummed theory works up to $G=10$, and in this range the bias increases with increasing $G$. The resummed theory also predicts that the moments increase as St increases, for fixed $G$. A more detailed analysis of the recursion leading to Eq. (6) reveals, however, that the limit $G \rightarrow \infty$ is delicate. Perfect alignment requires $\lambda=\infty$ [33].
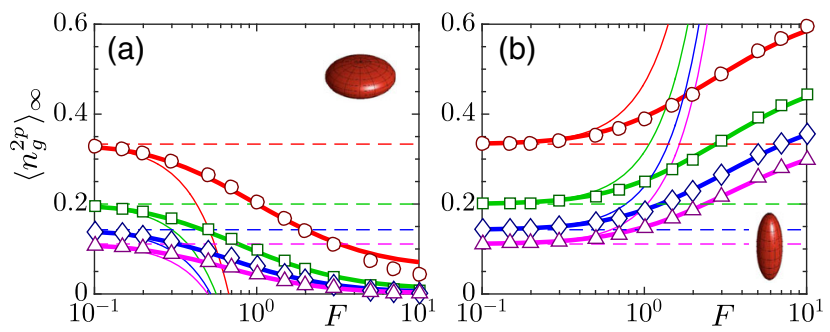

FIG. 2. Moments of $n_{g}=|\boldsymbol{n} \cdot \hat{\boldsymbol{g}}|$, for $p=1$ (circle), $p=2$ (square), $p=3$ (diamond), and $p=4$ (triangle). Dashed lines: moments of isotropic orientation distribution. Thin solid lines: Eq. (6) to $O\left(G^{2}\right)$. Thick solid lines: order 8-by-8 Padé-Borel resummation of Eq. (6) to $O\left(G^{32}\right)$. Parameters: $\mathrm{Ku}=0.1$, $\mathrm{St}=10$, with (a) $\lambda^{2}=0.1$ or (b) $\lambda^{2}=10$. 

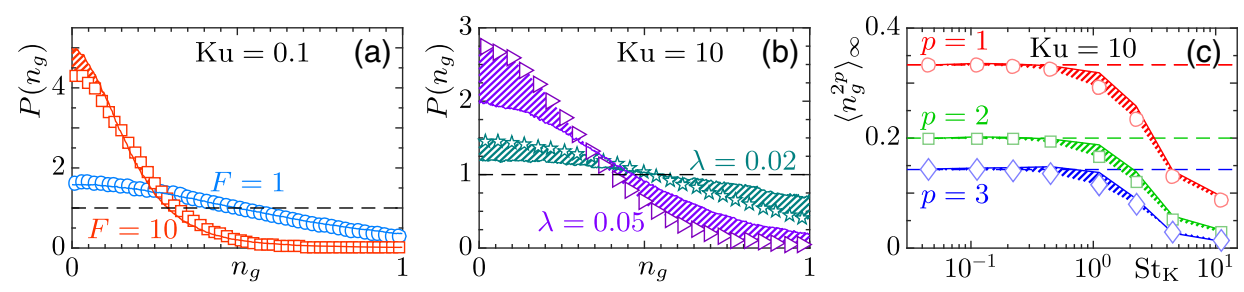

FIG. 3. History effect causes orientation bias. (a) Distribution of $n_{g}$ based on full statistical-model simulations (symbols), and based on straight deterministic paths (solid lines). Parameters: $\lambda=1 / \sqrt{10}, \mathrm{Ku}=0.1, \mathrm{St}=10, F=1$ (circle), $F=10$ (square). (b) Same for parameters corresponding to data in Fig. 1(a), $\lambda=0.02$ (star), $\lambda=0.05$ (right-pointing triangle). Preferential-sampling contribution is hatched. (c) Moments $\left\langle n_{g}^{2 p}\right\rangle_{\infty}$ from statistical-model simulations in the persistent limit (Ku $\left.=10\right)$ against $\operatorname{St}_{\mathrm{K}}(p=1$, circle; $p=2$, square; $p=3$, diamond). Parameters $\lambda=1 / \sqrt{10}, F_{\mathrm{K}} \approx 2.5$. Also shown are simulations based on straight deterministic paths (solid lines).

In summary, perturbation theory in $\mathrm{Ku}$ shows that turbulence gives rise to an orientation bias (Fig. 2), in excellent agreement with statistical-model simulations at $\mathrm{Ku}=0.1$ and in qualitative agreement with numerical results based on DNS (Fig. 1).

The calculations leading to Eq. (6) reveal that each moment $\left\langle\left(\boldsymbol{n}_{t} \cdot \hat{\boldsymbol{g}}\right)^{2 p}\right\rangle_{\infty}$ is a sum of two contributions that stem from the "preferential sampling" and "history" terms, defined above in connection with Eq. (4). For small Ku the history effect is dominant; the orientation bias is entirely determined by the history of fluid-velocity gradients along straight deterministic paths. Decomposing the leading-order contribution as $A_{1}^{(2)}=A_{1, \text { pref }}^{(2)}+A_{1 \text {,hist }}^{(2)}$ we find that $\left|A_{1, \text { pref }}^{(2)}\right| \ll$ $\left|A_{1, \text { hist }}^{(2)}\right|$ (Fig. S2(b) in the SM [33]). Figure 3(a) leads to the same conclusion. It shows the distribution $P\left(n_{g}\right)$ from statistical-model simulations for $\mathrm{Ku}=0.1$. Also shown is $P\left(n_{g}\right)$ computed for particles falling with constant velocity $\boldsymbol{v}=\boldsymbol{v}_{\mathrm{s}}\left(\boldsymbol{n}_{0}\right)$. In these simulations we choose the initial condition $\boldsymbol{n}_{0}$ so that $\boldsymbol{n}_{0} \boldsymbol{n}_{0}^{\top}$ in Eq. (3) equals the steady-state average $\left\langle\boldsymbol{n}_{0} \boldsymbol{n}_{0}^{\top}\right\rangle_{\infty}$, evaluated using the small-Ku theory. This corresponds to keeping just the history contribution to $\left\langle n_{g}^{2 p}\right\rangle_{\infty}$. We observe excellent agreement with the full statistical-model simulations. This shows that the history effect causes the orientation bias at small $\mathrm{Ku}$.

Persistent limit.-In the persistent limit we use numerical statistical-model simulations with $\mathrm{Ku}=10$ to analyze the orientation bias in the same way as for small $\mathrm{Ku}$. The result is shown in Fig. 3(b) [data represented by symbols is identical to the data for the two largest aspect ratios in Fig. 1(a)]. We plot the full statistical-model distribution and results for particles with a constant velocity (3) (neglecting preferential sampling). Here the average $\left\langle\boldsymbol{n}_{0} \boldsymbol{n}_{0}^{\top}\right\rangle_{\infty}$ in Eq. (3) is computed using statistical-model simulations. We infer that the history effect makes a substantial contribution to $P\left(n_{g}\right)$. But since the distributions do not match, we infer that preferential sampling also contributes. This contribution is hatched in Fig. 3(b).

Limit of large settling speeds.-Figure 3(c) shows statistical-model results for the moments $\left\langle n_{g}^{2 p}\right\rangle_{\infty}$ for $p \leq 3$ in the persistent limit, as functions of $\mathrm{St}_{\mathrm{K}}$. Open symbols denote full statistical-model simulations, and solid lines correspond to simulations based on straight deterministic paths. The difference between the two simulations demonstrates the importance of preferential sampling at intermediate $\mathrm{St}_{\mathrm{K}}$. As the Stokes number grows, however, the figure demonstrates that preferential sampling ceases to play a role. In this limit the orientation bias is entirely caused by the history effect. The bias shown in Fig. 3(c) increases as $\mathrm{St}_{\mathrm{K}}$ increases. However, as the perturbation theory indicates, the limit of large settling speeds is quite subtle. Statistical-model simulations for $\mathrm{Ku}=1$ show that the degree of alignment starts to decrease for very large $G$.

Conclusions.-We analyzed a statistical model for the orientational dynamics of small heavy spheroids settling in turbulence. The predictions of the model agree well with our own numerical results based on DNS of homogeneous isotropic turbulence (Fig. 1). Our statistical-model analysis shows that there are two distinct competing mechanisms causing the orientation bias: preferential sampling and the history effect. The latter dominates for large settling speeds, but it also makes substantial contributions in other parameter regimes. Preferential sampling dominates only when the bias is negligibly small. When the bias is significant, the history effect explains at least about $50 \%$ of the bias observed in Fig. 1.

We have shown that the orientation alignment depends on combinations of dimensionless numbers $\mathrm{St}_{\mathrm{K}}$ and $F_{\mathrm{K}} \mathrm{Re}_{\lambda}^{-1 / 2}$. Our analysis shows that it is the small-scale properties of the flow that determine the orientation alignment. The $\operatorname{Re}_{\lambda}$ dependence arises only because it determines the ratio between the smooth scale $\ell$ to $\eta_{\mathrm{K}}$. We note that $F_{\mathrm{K}} \mathrm{Re}_{\lambda}^{-1 / 2}$ equals the ratio of the settling velocity and the rms turbulent velocity fluctuations.

Our results pertain to small ice crystals settling in turbulent clouds, and allow us to model the sensitive dependence of the effect upon particle shape, size, and the turbulence intensity. This is important since turbulent dissipation rates vary widely in clouds. Our results predict strongly varying degrees of alignment. That the statistical model is in excellent agreement with the DNS opens a way to parametrize the orientation distribution of ice crystals in clouds. This potentially leads to an improved understanding of the radiative properties of clouds, and of particle aggregation through collisions in clouds. 
The present work is based on the point-particle approximation of heavy particles, which neglects the effect of fluid inertia. This requires the particle Reynolds number $\operatorname{Re}_{p} \equiv a v_{c} / \nu$ to be small, where $a=\max \left(a_{\|}, a_{\perp}\right)$. Estimating the slip velocity $v_{c}$ by the Stokes settling speed, we find that $\operatorname{Re}_{p}$ is of order unity for the data shown in Fig. 1, so the condition is marginally satisfied. The shear Reynolds number, $\operatorname{Re}_{s}$, must also be small. Since $\operatorname{Re}_{s} \equiv$ $a^{2} \sqrt{\left\langle\operatorname{tr} \mathbb{S}^{2}\right\rangle} / \nu \sim\left(a / \eta_{\mathrm{K}}\right)^{2}$ [45], this condition is satisfied for small particles.

Lopez et al. [46] analyzed the orientational dynamics of rods settling in a vortical flow. For small $\operatorname{Re}_{p}$ they found a bimodal distribution, with peaks at $n_{g}=0$ and 1 . They explain the peak at $n_{g}=0$ by the effect of fluid inertia. Our theory may explain the peak at $n_{g}=1$. These results point to a competition between turbulent and fluid-inertia torques, which remains to be understood. For small St one may formulate an ad hoc model by simply adding turbulent and fluid-inertia torques, along the lines suggested in Ref. [46]. But in general it remains a challenge to take into account effects due to fluid inertia from first principles, in a turbulent environment. Simulations resolving particle and fluid motion [47,48] and experiments [49-53] for micron-sized particles in turbulence are needed to test the predictions, and to determine the orientational dynamics of larger particles where fluid inertia must matter [53]. Finally, how to extend the ideas developed here to particles lighter than the fluid remains a challenging task.

This work was supported by Vetenskapsrådet (Grant No. 2013-3992), Formas (Grant No. 2014-585), and by the grant "Bottlenecks for particle growth in turbulent aerosols" from the Knut and Alice Wallenberg Foundation, Grant No. Dnr. KAW 2014.0048. J. J. and A. P. acknowledge the support of the Alexander von Humboldt Foundation. The numerical computations used resources provided by $\mathrm{C} 3 \mathrm{SE}$ and SNIC, and by the PSMN at ENS de Lyon.

[1] H. R. Pruppacher and J. D. Klett, Microphysics of Clouds and Precipitation, 2nd ed. (Kluwer Academic Publishers, Dordrecht, The Netherlands, 1997).

[2] H.-R. Cho, J. V. Iribarne, and W. G. Richards, On the orientation of ice crystals in a cumulonimubs cloud, J. Atmos. Sci. 38, 1111 (1981).

[3] J. P. Chen and D. Lamb, The theoretical basis for the parmaetrization of ice crystal habits: Growth by vapor deposition, J. Atmos. Sci. 51, 1206 (1994).

[4] J. C. Hubbert, S. M. Ellis, W. Y. Change, S. Rutledge, and M. Dixon, Modeling and interpretation of s-band ice crystal depolarization signatures from data obtained by simultaneously transmitting horizontally and vertically polarized fields, J. Appl. Meteor. Climatol. 53, 1659 (2014).

[5] J. Ruiz, D Macas, and F. Peters, Turbulence increases the average settling velocity of phytoplankton cells, Proc. Natl. Acad. Sci. U.S.A. 101, 17720 (2004).
[6] W. M. Durham, E. Climent, M. Barry, F. De Lillo, G. Boffetta, M. Cencini, and R. Stocker, Turbulence drives microscale patches of motile phytoplankton, Nat. Commun. 4, 2148 (2013).

[7] K. Gustavsson, F. Berglund, P. R. Jonsson, and B. Mehlig, Preferential Sampling and Small-Scale Clustering of Gyrotactic Microswimmers in Turbulence, Phys. Rev. Lett. 116, 108104 (2016).

[8] J. S. Guasto, R. Rusconi, and R. Stocker, Fluid mechanics of planktonic microorganisms, Annu. Rev. Fluid Mech. 44, 373 (2012).

[9] P. Yang, K. N. Liou, L. Bin, C. Liu, B. Yi, and B. A. Baum, On the radiative properties of ice clouds: Light scattering, remote sensing and radiation parametrization, Adv. Atmos. Sci. 32, 32 (2015).

[10] M. R. Maxey, The gravitational settling of aerosol particles in homogeneous turbulence and random flow fields, J. Fluid Mech. 174, 441 (1987).

[11] G. H. Good, P. J. Ireland, G. Bewley, E. Bodenschatz, L. R. Collins, and Z. Warhaft, Settling regimes of inertial particles in isotropic turbulence, J. Fluid Mech. 759, R3 (2014).

[12] K. Gustavsson, S. Vajedi, and B. Mehlig, Clustering of Particles Falling in a Turbulent Flow, Phys. Rev. Lett. 112, 214501 (2014).

[13] J. Bec, H. Homann, and S. S. Ray, Gravity-Driven Enhancement of Heavy Particle Clustering in Turbulent Flow, Phys. Rev. Lett. 112, 184501 (2014).

[14] P. J. Ireland, A. D. Bragg, and L. R. Collins, The effect of Reynolds number on inertial particle dynamics in isotropic turbulence. Part 2. Simulations with gravitational effects, J. Fluid Mech. 796, 659 (2016).

[15] V. Mathai, E. Calzavarini, J. Brons, C. Sun, and D. Lohse, Microbubbles and Microparticles Are Not Faithful Tracers of Turbulent Acceleration, Phys. Rev. Lett. 117, 024501 (2016).

[16] H. Parishani, O. Ayala, B. Rosa, L.-P. Wang, and W. W. Grabowski, Effects of gravity on the acceleration and pair statistics of inertial particles in homogeneous isotropic turbulence, Phys. Fluids 27, 033304 (2015).

[17] R. E. Khayat and R. G. Cox, Inertia effects on the motion of long slender bodies, J. Fluid Mech. 209, 435 (1989).

[18] V. Dabade, K. M. Navaneeth, and G. Subramanian, Effects of inertia and viscoelasticity on sedimenting anisotropic particles, J. Fluid Mech. 778, 133 (2015).

[19] F. Candelier and B. Mehlig, Settling of an asymmetric dumbbell in a quiescent fluid, J. Fluid Mech. 802, 174 (2016).

[20] A. Roy, L. Tierney, G. A. Voth, and D. L. Koch, Inertial symmetry-breaking transitions in the settling of asymmetric rod-like and ramified particles, 2016.

[21] M. N. Ardekani, P. Costa, W. P. Breugem, and L. Brandt, Numerical study of the sedimentation of spheroidal particles, Int. J. Multiphase Flow 87, 16 (2016).

[22] G. B. Jeffery, The motion of ellipsoidal particles immersed in a viscous fluid, Proc. R. Soc. A 102, 161 (1922).

[23] A. Pumir and M. Wilkinson, Orientation statistics of small particles in turbulence, New J. Phys. 13, 093030 (2011).

[24] S. Parsa, E. Calzavarini, F. Toschi, and G. A. Voth, Rotation Rate of Rods in Turbulent Fluid Flow, Phys. Rev. Lett. 109, 134501 (2012). 
[25] L. Chevillard and C. Meneveau, Orientation dynamics of small, triaxial-ellipsoidal particles in isotropic turbulence, J. Fluid Mech. 737, 571 (2013).

[26] K. Gustavsson, J. Einarsson, and B. Mehlig, Tumbling of Small Axisymmetric Particles in Random and Turbulent Flows, Phys. Rev. Lett. 112, 014501 (2014).

[27] M. Byron, J. Einarsson, K. Gustavsson, G. A. Voth, B. Mehlig, and E. Variano, Shape-dependence of particle rotation in isotropic turbulence, Phys. Fluids 27, 035101 (2015).

[28] L. Zhao, N. R. Challabotla, H. I. Andersson, and E. A. Variano, Rotation of Nonspherical Particles in Turbulent Channel Flow, Phys. Rev. Lett. 115, 244501 (2015).

[29] G. Voth, Disks aligned in a turbulent channel, J. Fluid Mech. 772, 1 (2015).

[30] G. Voth and A. Soldati, Anisotropic particles in turbulence, Annu. Rev. Fluid Mech. 49, 249 (2017).

[31] C. Siewert, R. P. J. Kunnen, M. Meinke, and W. Schröder, Orientation statistics and settling velocity of ellipsoids in decaying turbulence, Atmos. Res. 142, 45 (2014).

[32] J. Schumacher, J. D. Scheel, D. Krasnov, D. A. Donzis, V. Yakhot, and K. R. Sreenivasan, Small-scale universality in fluid turbulence, Proc. Natl. Acad. Sci. U.S.A. 111, 10961 (2014).

[33] See Supplemental Material at http://link.aps.org/ supplemental/10.1103/PhysRevLett.119.254501 for details, which includes Refs. [34,35].

[34] L. D. Landau and E. M. Lifshitz, Mechanics (Pergamon Press Ltd., Oxford, 1969).

[35] S. A. Orszag, On the elimination of aliasing in finitedifference schemes by filtering high-wavenumber components, J. Atmos. Sci. 28, 1074 (1971).

[36] K. Gustavsson and B. Mehlig, Statistical models for spatial patterns of heavy particles in turbulence, Adv. Phys. 65, 1 (2016).

[37] S. Kim and S. J. Karrila, Microhydrodynamics: Principles and Selected Applications (Butterworth-Heinemann, Boston, 1991).

[38] C. Marchioli, M. Fantoni, and A. Soldati, Orientation, distribution, and deposition of elongated, inertial fibers in turbulent channel flow, Phys. Fluids 22, 033301 (2010).

[39] J. Fries, J. Einarsson, and B. Mehlig, Angular dynamics of small crystals in viscous flow, Phys. Rev. Fluids 2, 014302 (2017).
[40] M. Voßkuhle, A. Pumir, E. Lévêque, and M. Wilkinson, Prevalence of the sling effect for enhancing collision rates in turbulent suspensions, J. Fluid Mech. 749, 841 (2014).

[41] W. W. Grabowski and P. Vailancourt, Comments on preferential concentration of cloud droplets by turbulence: Effects on the early evolution of cumulus cloud droplet spectra, J. Atmos. Sci. 56, 1433 (1999).

[42] E. Calzavarini, R. Volk, M. Bourgoin, E. Leveque, J.F. Pinton, and F. Toschi, Acceleration statistics of finite-sized particles in turbulent flow: The role of Faxén forces, J. Fluid Mech. 630, 179 (2009).

[43] K. Gustavsson and B. Mehlig, Ergodic and non-ergodic clustering of inertial particles, Europhys. Lett. 96, 60012 (2011).

[44] C. M. Bender and S. A. Orszag, Advanced Mathematical Methods for Scientists and Engineers (McGraw-Hill, New York, 1978).

[45] F. Candelier, J. Einarsson, and B. Mehlig, Rotation of a Small Particle in Turbulence, Phys. Rev. Lett. 117, 204501 (2016).

[46] D. Lopez and E. Guazzelli, Inertial effects on fibers settling in a vortical flow, Phys. Rev. Fluids 2, 024306 (2017).

[47] H. Homann and J. Bec, Finite-size effects in the dynamics of neutrally buoyant particles in turbulent flow, J. Fluid Mech. 651, 81 (2010).

[48] W. Fornari, F. Picano, G. Sardina, and L. Brandt, Reduced particle settling speed in turbulence (to be published).

[49] H. Traugott and A. Liberzon, Experimental study of forces on freely moving spherical particles during resuspension into turbulent flow, Int. J. Multiphase Flow 88, 167 (2017).

[50] V. Mathai, V. N. Prakash, J. Brons, C. Sun, and D. Lohse, Wake-Driven Dynamics of Finite-Sized Buoyant Spheres in Turbulence, Phys. Rev. Lett. 115, 124501 (2015).

[51] A. D. Bordoloi, and E. Variano, Rotational kinematics of large cylindrical particles in turbulence, J. Fluid Mech. 815, 199 (2017).

[52] G. G. Marcus, S. Parsa, S. Kramel, R. Ni, and G. A. Voth, Measurements of the solid-body rotation of anisotropic particles in 3D turbulence, New J. Phys. 16, 102001 (2014).

[53] S. Kramel, L. Tierney, W. Rees, G. A. Voth, U. Menon, A. Roy, and D. L. Koch, Orientation statistics of non-spherical particles sedimenting in turbulence, in 69th Annual Meeting of the APS Division of Fluid Dynamics, Portland, Oregon (2016), Abstract No. E28.00002. 